\title{
Uniqueness of infrared asymptotics in Landau gauge Yang-Mills theory II
}

\author{
Christian S. Fischer ${ }^{1,2}$ and Jan M. Pawlowski ${ }^{3,4}$ \\ ${ }^{1}$ Institut für Kernphysik, Technische Universität Darmstadt, Schlossgartenstraße 9, \\ D-64289 Darmstadt, Germany \\ ${ }^{2}$ GSI Helmholtzzentrum für Schwerionenforschung GmbH, Planckstr. 1 D-64291 Darmstadt, Germany. \\ ${ }^{3}$ Institut für Theoretische Physik, University of Heidelberg, \\ Philosophenweg 16, D-62910 Heidelberg, Germany. \\ ${ }^{4}$ ExtreMe Matter Institute EMMI, GSI Helmholtzzentrum für \\ Schwerionenforschung mbH, Planckstr. 1 D-64291 Darmstadt, Germany.
}

\begin{abstract}
We present a shortened and simplified version of our proof [8] of the uniqueness of the scaling solution for the infrared asymptotics of Green functions in Landau gauge Yang-Mills theory. The simplification relates to a new RG-invariant arrangement of Green functions applicable to general theories. As before the proof relies on the necessary consistency between Dyson-Schwinger equations (DSEs) and functional renormalisation group equations (FRGs). We also demonstrate the existence of a specific scaling solution for both, DSEs and FRGs, that displays uniform and soft kinematic singularities.
\end{abstract}

PACS numbers: 12.38.Aw,11.15.Tk,05.10.Cc,02.30.Rz

\section{INTRODUCTION}

The infrared behaviour of strongly interacting quantum field theories is of general interest. In particular the infrared behaviour of Landau gauge Yang-Mills theory is a much debated issue in the past years. This is due to the close connection of the ghost and gluon propagators to confinement scenarios proposed by Kugo and Ojima 1], Gribov [2] and Zwanziger [3]. Within functional methods a self-consistent infrared asymptotic solution of the whole tower of Dyson-Schwinger equations (DSEs) and functional renormalisation group equations (FRGs) has been found [4, 5, 6, 7, 8, 9] that supports these scenarios and is also consistent with global BRST symmetry [10]. This scaling solution implies that all Greens functions scale like a power of momentum in the infrared with interrelated anomalous dimensions if all momenta are scaled. That entails the absence of mass scales below which some degrees of freedom decouple. In such a scenario the scaling power of Green functions can be extracted by a power counting analysis. As a further direct consequence all couplings have fixed points at zero momentum.

An alternative infrared solution of Yang-Mills theory is provided by the decoupling solution [11, 12, 13, 14]. This type of solutions has been discussed in detail in [10], and has been shown to be inconsistent with global BRST symmetry. In the present context it can be interpreted as the decoupling of (part of) the propagating degrees of freedom below a mass scale. In such a case the infrared asymptotics cannot be fixed uniquely by a scaling analysis.

In [8] we suggested a combined analysis of the towers of DSEs and FRGs for an infrared scaling analysis, being applicable to general theories. We have shown that apart from decoupling there is only one, unique scaling solution of infrared Yang-Mills theory in Landau gauge. Here we present a greatly simplified version of our proof which also allows the reduction of the number of presuppositions. The result, of course, is the same as in 7, 8, 9]. In addition we present an explicit scaling solution for DSEs and FRGs that involves also kinematical singularities [15]. The knowledge of these kinematical singularities is not necessary for the proof of the existence and uniqueness of the global scaling, and were not discussed explicitly in our previous work [8].

In Section $\amalg$ we introduce FRG and DSE equations for the effective action and discuss momentum and RG scaling for the effective action and its vertices. With help of Appendix A the functional equations are written in a similar form. A convenient parameterisation of the vertices is introduced that splits off the renormalisation group (RG)-scaling and reduces the discussion to that of the scaling properties of RG-invariant quantities. This very natural reduction is the key ingredient of the simplification of the proof, and is applicable to general theories. It also allows us to provide heuristic arguments for the existence of a unique scaling solution, that should facilitate the following of the proof. In Section [II] we derive the respective scaling constraints from FRG and DSE equations, the combination of which provides a unique scaling solution. In Section IV we extend our analysis to kinematical singularities of vertices, the details can be found in Appendix B. We close with a short summary of our findings.

\section{FUNCTIONAL RELATIONS FOR THE EFFECTIVE ACTION}

The starting point of our analysis is the functional form of FRGs and DSEs, depicted in Fig. 1 and Fig. 2 respectively. In Fig. 1 we have rewritten the FRG in a form similar to the DSEs in Fig. 2, This is detailed in Appendix A. It is well-known from the evaluation of critical physics that the DSE is less amiable to the dis- 


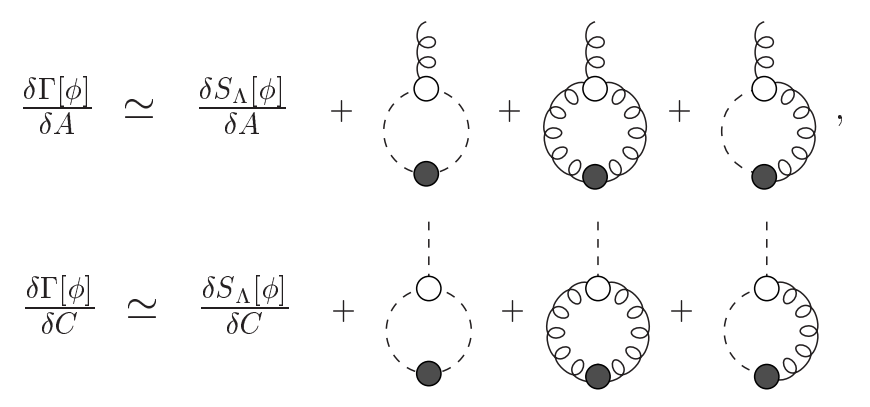

FIG. 1: Infrared asymptotics of the FRG. Filled circles denote fully dressed field dependent propagators. Empty circles denote fully dressed field dependent vertices.

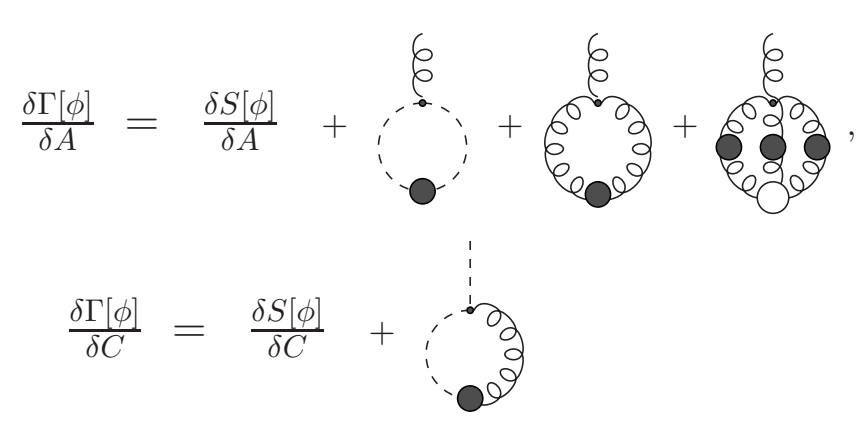

FIG. 2: Functional Dyson-Schwinger equation (DSE) for the effective action. Filled circles denote fully dressed field dependent propagators. Empty circles denote fully dressed field dependent vertices, dots denote field dependent bare vertices.

cussion of scaling than the FRG. In general scaling in the DSE only comes from a combination of diagrams which hosts cancellations effectively leading to the substitution of the bare vertex present in each diagram with dressed ones. In general such a cancellation also includes the classical term. One example for such cancellations is the $\phi^{4}$-theory.

In the present case, however, the functional ghost DSE (second line of Fig. 2) prohibits cancellations between diagrams as it only consists of one diagram. It is for this reason that the DSE system of Yang-Mills theory cannot be subject to cancellations except for kinematical and global symmetry reasons. Moreover, in both sets of equations Fig. [1 Fig. 2 we have the classical term in the DSE or the initial condition for the FRG $\delta S_{(\Lambda)} / \delta \phi$. For infrared enhanced vertices and inverse propagators this term is subleading. On the other hand, scaling of infrared suppressed vertices and inverse propagators requires cancellations on the right hand side of the FRGs and DSEs between the diagrams and the classical term. As will become clear later the only place where such a cancellation necessarily has to occur for a scaling solution are the DSE and FRG of the ghost propagator. The presence or absence of cancellations in these equations therefore decides about the existence of the scaling solution, and is related to global properties of the gauge fix- ing, namely the Kugo-Ojima confinement criterion and the Gribov-Zwanziger horizon condition, see also [10].

The functional DSEs and FRGs are derived from the effective action of the theory, expanded in its Green functions. With the abbreviation

$$
\int_{p_{1} \cdots p_{l}} \equiv \int \prod_{i=1}^{l}\left(\frac{d^{d} p_{i}}{(2 \pi)^{d}}\right)(2 \pi)^{d} \delta^{d}\left(\sum_{j=1}^{l} p_{j}\right) .
$$

the effective action is given as

$$
\begin{aligned}
\Gamma[\phi]= & \sum_{m, n} \frac{1}{m !(n !)^{2}} \int_{p_{1} \cdots p_{2 n+m}} \Gamma^{(2 n, m)}\left(p_{1} \cdots p_{2 n+m}\right) \\
& \times \prod_{i=1}^{n} \bar{C}\left(p_{i}\right) \prod_{i=n+1}^{2 n} C\left(p_{i}\right) \prod_{i=2 n+1}^{2 n+m} A\left(p_{i}\right) \\
\equiv & \sum_{m, n} \frac{1}{m !(n !)^{2}} \Gamma^{(2 n, m)} \bar{C}^{n} C^{n} A^{m}
\end{aligned}
$$

with the gluon field $A$ and the (anti-)ghost fields $\bar{C}, C$, $\phi=(A, \bar{C}, C)$ and suppressed Lorentz- and colourindices. In the third line in Eq.(2) we have introduced an abbreviated notation which will be used throughout this work. The one-particle-irreducible Greens functions $\Gamma^{(2 n, m)}$ with $2 n$ external (anti-)ghost legs and $m$ external gluon legs are the expansion coefficients of the effective action in the field expansion. It is convenient to reparameterise these expansion coefficients with the help of the coefficients

$$
\Gamma^{(2,0)} \equiv Z^{(2,0)} S_{\mathrm{cl}}^{(2,0)}, \quad \Gamma^{(0,2)} \equiv Z^{(0,2)} S_{\mathrm{cl}}^{(0,2)}
$$

of the kinetic terms. We then obtain the rescaled coefficients $\bar{\Gamma}^{(2 n, m)}(\vec{p})$ given by

$$
\begin{aligned}
\Gamma^{(2 n, m)}(\vec{p})= & \bar{\Gamma}^{(2 n, m)}(\vec{p}) \\
& \times \prod_{i=1}^{2 n} \sqrt{Z^{(2,0)}\left(p_{i}\right)} \prod_{i=1}^{m} \sqrt{Z^{(0,2)}\left(p_{2 n+i}\right)} .
\end{aligned}
$$

with $\vec{p}=\left(p_{1}, \ldots, p_{2 n+m}\right)$. This parameterisation implies that the coefficients of the two point Green functions

$$
\bar{\Gamma}^{(2,0)}(p)=S_{\mathrm{cl}}^{(2,0)}(p), \quad \bar{\Gamma}^{(0,2)}(p)=S_{\mathrm{cl}}^{(0,2)}(p),
$$

carry only the canonical momentum dependence of the kinetic terms and $Z^{(0,2)}, Z^{(2,0)}$ account for all quantum corrections.

The reparametrisation (4) also entails that the $Z$ factors on the right hand side of (4) carry the whole renormalisation group scaling of the vertex functions $\Gamma^{(2 n, m)}$ in terms of the renormalisation scale $\mu$. Together with the standard renormalisation group (RG) equation of the theory,

$$
\mu \frac{d}{d \mu} \Gamma=0,
$$


we then learn from (4) and (6) that the expansion coefficients $\bar{\Gamma}^{(2 n, m)}$ do not depend on $\mu$, i.e.

$$
\mu \frac{d}{d \mu} \bar{\Gamma}^{(2 n, m)}=0, \quad \forall n, m \in \mathbb{Z} .
$$

Note in this context that (6) is also valid in the presence of the RG-adapted regulator terms in the functional renormalisation group equations (FRGs) [16, 17]. This allows us to derive constraints for the vertex functions $\bar{\Gamma}^{(2 n, m)}$ also in the presence of the regulator, see [6, 8$]$ for details. In the present work we also show, see Appendix $\mathrm{A}$, that the FRG-analysis of the IR-asymptotics can be further simplified, allowing for a more direct approach.

Eq. (77) already suggests that the RG-invariant coefficients $\bar{\Gamma}^{(2 n, m)}$ do not carry any (global) anomalous scaling in terms of momenta. We shall show in the following that this is indeed the case.

\section{UNIQUENESS OF THE GLOBAL SCALING}

For this proof we are only interested in the global scaling behaviour for the coefficient functions $\bar{\Gamma}^{(2 n, m)}$. Modulo logarithms this entails the global scaling

$$
\lim _{\lambda \rightarrow 0} \bar{\Gamma}^{(2 n, m)}(\lambda \vec{p})=\lambda^{2\left(d_{2 n+m}+\bar{\kappa}_{2 n, m}\right)} \bar{\Gamma}_{\mathrm{as}}^{(2 n, m)}(\vec{p}),
$$

where $\bar{\Gamma}_{\text {as }}$ stands for the infrared leading term, and $\vec{p}=$ $\left(p_{1}, \ldots, p_{2 n+m}\right)$. The coefficient

$$
d_{l}=\frac{d}{2}-l \frac{d-2}{4},
$$

is the canonical scaling dimension of the vertex $\Gamma^{(2 n, m)}$ with $l=2 n+m$. Note that it is only sensitive to the total number of external legs. It can be directly derived from Eq. (2): plugged into Eq. (2) it matches the canonical scaling of the $\delta$-function of total momentum conservation, $-d / 2$, and that of ghost, anti-ghost and gluon fields in position space, $(d-2) / 4$. Thus it matches the momentum scaling of the momentum integral $\int_{p_{1} \cdots p_{2 n+m}}$ and that of the fields in momentum space. As a result the scaling (9) includes the canonical momentum scaling of the oneparticle irreducible Green functions as well as the scaling of the couplings. Hence, only in the critical dimension of Yang-Mills theory, $d=4$, the canonical scaling dimension (9) agrees with the classical momentum scaling.

It turns out that the present parameterisation (8) in terms of $\bar{\kappa}_{2 n, m}$ enables us to significantly simplify the proof given in Ref. [8] of the uniqueness of the $\kappa_{2 n, m}$. At its core the reason is the natural book-keeping of the necessary RG-scaling by the $Z$-factors that incorporate one factor of $1 / 2 \kappa_{2,0}$ or $1 / 2 \kappa_{0,2}$ for each external leg of the vertex $\Gamma^{(2 n, m)}$.

As for the most basic $\bar{\kappa}_{2 n, m}$ we obtain by definition (cp. Eq. (5))

$$
\bar{\kappa}_{2,0}=\bar{\kappa}_{0,2}=0 \text {. }
$$

Then the scaling relations for the kinetic terms, the ghost and gluon dressing functions, read

$$
\begin{aligned}
& \lim _{\lambda \rightarrow 0} Z^{(2,0)}(\lambda p)=\lambda^{\kappa_{2,0}} Z^{(2,0)}(p), \\
& \lim _{\lambda \rightarrow 0} Z^{(0,2)}(\lambda p)=\lambda^{\kappa_{0,2}} Z^{(0,2)}(p) .
\end{aligned}
$$

The total global scaling $\lambda^{2 t_{2 n, m}}$ of the full vertices $\Gamma^{(2 n, m)}$ also involves the anomalous dimensions of the propagators and reads

$$
t_{2 n, m}=d_{2 n+m}+\frac{1}{2}\left(2 n \kappa_{2,0}+m \kappa_{0,2}\right)+\bar{\kappa}_{2 n, m} .
$$

Previous analyses in [7, 8, 9, 15] were initiated similarly. However, instead of evaluating the deviation $\bar{\kappa}_{2 n, m}$ to the standard anomalous scaling, the deviation $\kappa_{2 n, m}$ from the canonical scaling in the critical dimension four of YangMills theory was evaluated,

$$
t_{2 n, m}=\left.d_{2 n, m}\right|_{d=4}+\kappa_{2 n, m},
$$

where

$$
\kappa_{2 n, m}=\Delta d_{2 n+m}+\frac{1}{2}\left(2 n \kappa_{2,0}+m \kappa_{0,2}\right)+\bar{\kappa}_{2 n, m},
$$

with the deviation $\Delta d_{2 n+m}$ of the canonical scaling from that in the critical dimension four,

$$
\Delta d_{l}=d_{l}-\left.d_{l}\right|_{d=4}=(2-l) \frac{d-4}{4}
$$

and $l=2 n+m$. This is adapted such that the $\kappa_{2 n, m}$ for primitively divergent vertices describe the full scaling of the corresponding dressing functions in $d$ dimensions.

We emphasise that in principle additional logarithmic scalings should be included into (8), (11). However, even if present, additional logarithmic scalings do not change the relations between the $\bar{\kappa}_{n, m}$ and are therefore irrelevant for the purpose of the present investigation. We also add that self-consistent logarithmic scaling laws have to satisfy additional consistency conditions.

In four dimensions an explicit solution of the whole tower of DSEs and FRGs in terms of $\kappa_{2 n, m}$ was first derived in [7] and then generalised to $d$ dimensions in [9] It reads

$$
\begin{aligned}
\kappa & \equiv \kappa_{2,0}=-\frac{4-d}{4}-\frac{1}{2} \kappa_{0,2} \\
\kappa_{2 n, m} & =(n-m) \kappa+(1-n)\left(\frac{d}{2}-2\right) .
\end{aligned}
$$

In 8] we already gave a proof for the uniqueness of Eq. (16). In the following we reformulate this proof in a, to our mind, more elegant and instructive manner that also allows us to reduce the number of presuppositions. We first derive constraints for $\bar{\kappa}_{2 n, m}$ from the functional renormalisation group and Dyson-Schwinger equations and then show that $\bar{\kappa}_{2 n, m}=0$ for all $n, m$. The resulting expression for the $\kappa_{2 n, m}$ from Eq. (14) then agrees with Eq. (16). 


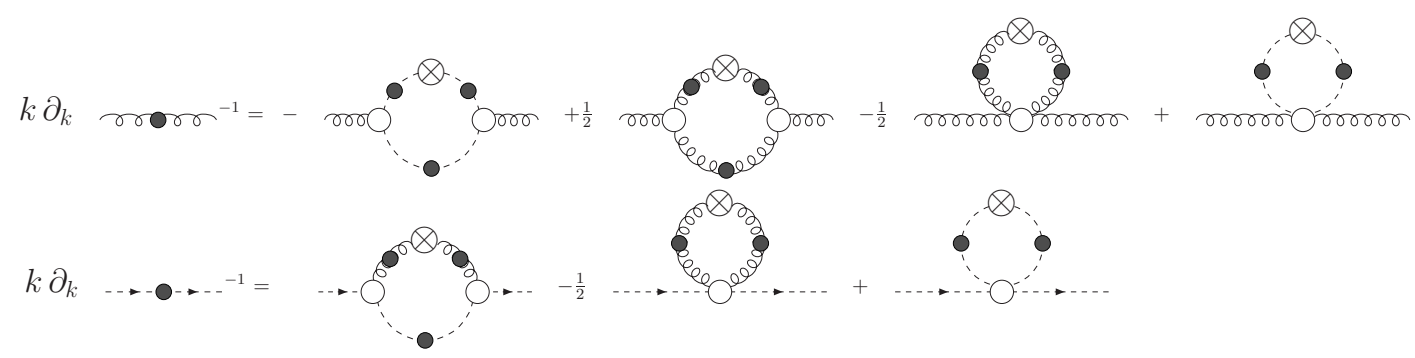

FIG. 3: Functional renormalisation group equations for the gluon and ghost propagator. Filled circles denote dressed propagators and empty circles denote dressed vertex functions. Crosses indicate insertions of the infrared cutoff function. Only one possible insertion of the infrared cutoff function per diagram is shown.

\section{A. Constraints from the functional RG}

The FRGs for the ghost and gluon propagators are given diagrammatically in Fig. 3. With a mode cut-off, which only removes a single momentum mode, the regulator insertion is proportional to a $\delta$-function and simply restricts the loop integral to a given momentum $p^{2}$ which we take to be vanishing. Then the loops on the rhs of the flow simply count the powers of global momentum scaling of the quantum fluctuations, no initial condition, similar to the classical term in the DSE, appears, see also [8]. The potential cancellations necessary for the initial condition are discussed at the end of our proof.

We are now counting anomalous dimensions on both sides of the equations in terms of powers of one external momentum scale $p^{2}$ in the infrared region $p^{2} \ll \Lambda_{Q C D}^{2}$. For the global scaling (8) considered here all anomalous dimensions in terms of internal momenta of the loops translate directly into anomalous dimensions of the external momentum scale. This is also true for the vertex equations considered below. In this respect the regulator insertion, denoted by the crosses, carry the anomalous dimensions of inverse propagators [6, 8].

The constraint equations for $\bar{\kappa}_{2 n, m}$ can be derived in several ways. A somewhat pedestrian approach is to count anomalous dimensions $\kappa_{2 n, m}$ of the dressing functions on both sides of the equations and then converting to $\bar{\kappa}_{2 n, m}$ with the help of (14). More efficiently, we note that the $\kappa_{2,0}, \kappa_{0,2}$ carry the renormalisation group scaling of the corresponding Green functions and match on both sides of the FRG equations. In particular this is true for the propagator FRGs in Fig. 3. Consequently all $\kappa_{2,0}, \kappa_{0,2}$ drop out of the FRG-relations for a general vertex $\Gamma_{2 n, m}$. Note also that the sum of the canonical dimensions $d_{2 i, j}$, (9), in a given diagram for $\Gamma_{2 n, m}$ simply gives the total canonical dimension $d_{2 n+m}$, and hence the $d_{2 i, j}$ also drop out of the FRG-relations. Then, we are left with relations for solely the $\bar{\kappa}_{2 i, j}$. For the propagators the constraints read

$$
\begin{aligned}
& 0=\bar{\kappa}_{0,2}=\min \left(2 \bar{\kappa}_{2,1}, 2 \bar{\kappa}_{0,3}, \bar{\kappa}_{0,4}, \bar{\kappa}_{2,2}\right), \\
& 0=\bar{\kappa}_{2,0}=\min \left(2 \bar{\kappa}_{2,1}, \bar{\kappa}_{2,2}, \bar{\kappa}_{4,0}\right),
\end{aligned}
$$

from the gluon and ghost FRGs. For the lhs of these equations we used that $\bar{\kappa}_{2,0}=\bar{\kappa}_{0,2}=0$ by definition, cp. Eq. (10). The minimum prescription on the right hand side of (17), (18) takes into account that only one of the diagrams may be leading in the infrared. The constraint (18) from the ghost-FRG entails

$$
\bar{\kappa}_{2,1} \geq 0, \quad \bar{\kappa}_{2,2} \geq 0, \quad \bar{\kappa}_{4,0} \geq 0,
$$

and at least one of these has to be zero for (18) to be satisfied,

$$
\bar{\kappa}_{2,1}=0, \quad \text { or } \quad \bar{\kappa}_{2,2}=0, \quad \text { or } \quad \bar{\kappa}_{4,0}=0 .(20)
$$

The same analysis for (17) entails that $\bar{\kappa}_{2,1}, \bar{\kappa}_{0,3}, \bar{\kappa}_{0,4}, \bar{\kappa}_{2,2} \geq 0$ with at least one of them being zero. For the proof below, however, Eqs. (19) and (20) will be sufficient.

We conclude the FRG-analysis with a discussion of the FRG relations for general Green functions. Schematically these relations read

$$
\bar{\kappa}_{2 n, m}=\min \left(\bar{\kappa}_{2 n+2, m}, \bar{\kappa}_{2 n, m+2}, \ldots\right),
$$

where the first two terms are the tadpole contribution with ghost tadpole $\left(\bar{\kappa}_{2 n+2, m}\right)$, and a gluon tadpole $\left(\bar{\kappa}_{2 n, m+2}\right)$ respectively. The dots stand for other diagrams with at least two vertices. It follows that $\bar{\kappa}_{2 n, m}$ appears as the tadpole contribution in the relation for $\bar{\kappa}_{2 n-2, m}$ and $\bar{\kappa}_{2 n, m-2}$, and more generally

$$
\bar{\kappa}_{2(n-r), m-2 s} \leq \bar{\kappa}_{2 n, m},
$$

for all $r<n$ and $2 s<m$. This allows us to relate general $\bar{\kappa}_{2 n, m}$ to either $\bar{\kappa}_{2,1}$ for odd $m$ and $2 s=m-1$, or $\bar{\kappa}_{2,2}$ for even $m$ and $2 s=m-2$. Thus we have

$$
\bar{\kappa}_{2 n, m} \geq\left\{\begin{array}{ll}
\bar{\kappa}_{2,2} & \text { for } m \text { even } \\
\bar{\kappa}_{2,1} & \text { for } m \text { odd }
\end{array},\right.
$$

and we conclude with (19) that

$$
\bar{\kappa}_{2 n, m} \geq 0, \quad \forall n, m \in \mathbb{N},
$$

in general space-time dimension $d$. This constraint together with Eq. (20) will be important in what follows, as it summarises in a closed form the infinite number of constraints from higher diagrams. 


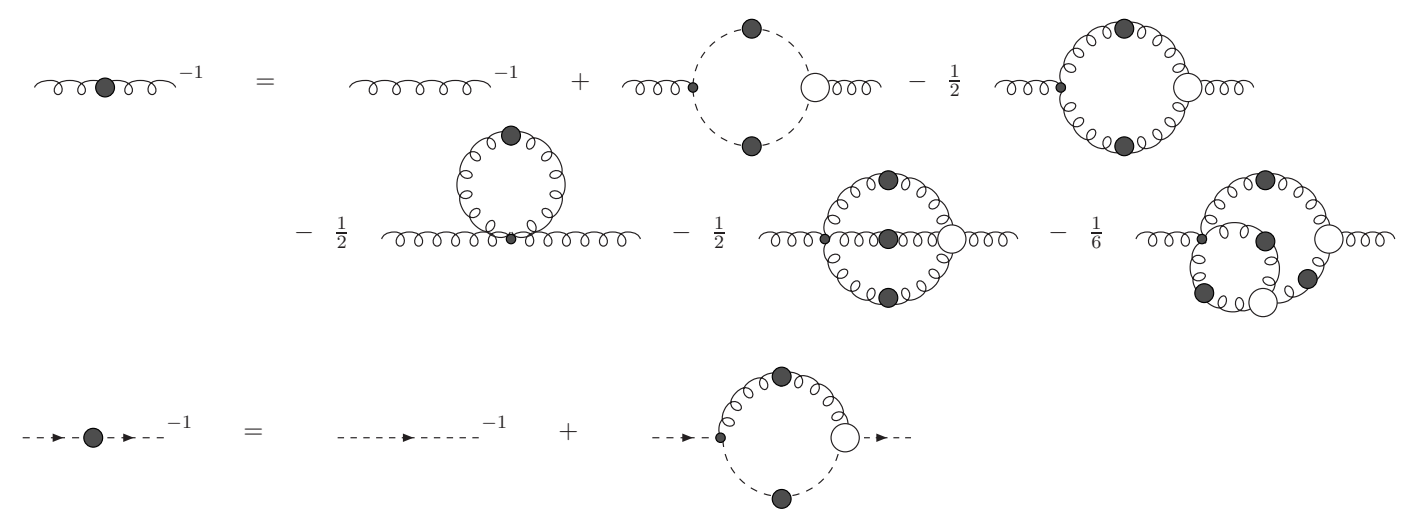

FIG. 4: Dyson-Schwinger equations for the gluon and ghost propagator. Filled circles denote dressed propagators and empty circles denote dressed vertex functions.

\section{B. Constraints from Dyson-Schwinger equations}

The Dyson-Schwinger equations for the ghost and gluon propagators are given diagrammatically in Fig. 4, whereas the corresponding equations for the ghost-gluon vertex are displayed in Fig. 5]. For the ghost gluon vertex we have two DSEs which are derived from either the functional gluon DSE or the functional ghost DSE, see [8]. As already mentioned, the potential cancellations necessary for the classical terms are discussed at the end of our proof.

We have seen in the analysis of the FRGs that the $\bar{\kappa}_{2 n, m}$-constraints boil down to simply counting the vertices involved in a given diagram and summing up the corresponding $\bar{\kappa}_{2 n, m}$. The same would apply to the DSEs if we only had dressed vertices in the DSE diagrams. However, there is always one bare vertex which then counts as $\bar{\kappa}_{2 n, m}-\kappa_{2 n, m} \equiv-\Delta \kappa_{2 n, m}$. These differences are given by

$$
\Delta \kappa_{2 n, m}=\Delta d_{2 n+m}+\frac{1}{2}\left(2 n \kappa_{2,0}+m \kappa_{0,2}\right) .
$$

For example, we are thus led to $\bar{\kappa}_{2,1}-\Delta \kappa_{2,1}$ for the right hand side of the ghost propagator DSE, and zero on the left hand side similar to the ghost FRG. This simple counting applies to all the diagrams. For its chief importance in the proof we introduce the abbreviation

$$
\Delta \kappa \equiv \Delta \kappa_{2,1}
$$

The constraints derived from the propagator DSEs displayed in Fig. 4 are then given by

$$
\begin{aligned}
0=\min \left(\bar{\kappa}_{2,1}-\Delta \kappa, \bar{\kappa}_{0,3}-\Delta \kappa_{0,3},-\Delta \kappa_{0,4},\right. \\
\left.\bar{\kappa}_{0,4}-\Delta \kappa_{0,4}, 2 \bar{\kappa}_{0,3}-\Delta \kappa_{0,4}\right), \\
0=\min \left(\bar{\kappa}_{2,1}-\Delta \kappa\right) .
\end{aligned}
$$

Certainly, these relations can be derived as well in the pedestrian way of counting $\kappa_{2 n, m}$ on both sides of the equations and converting them to $\bar{\kappa}_{2 n, m}$. Note that in contradistinction to the FRG equations the DSEs do depend on $\kappa_{2,0}$ and $\kappa_{0,2}$ via the $\Delta \kappa_{2 n, m}$.

In the two different DSEs for the ghost gluon vertex in Fig. 5 we again apply the now familiar counting and obtain

$\bar{\kappa}_{2,1}=\min \left(\bar{\kappa}_{2,1}+\bar{\kappa}_{0,3}-\Delta \kappa, 2 \bar{\kappa}_{2,1}-\Delta \kappa, \bar{\kappa}_{2,2}-\Delta \kappa\right)$,

from the upper relation in Fig. 5 and

$$
\begin{aligned}
\bar{\kappa}_{2,1}=\min \left(2 \bar{\kappa}_{2,1}-\Delta \kappa_{0,3}, 2 \bar{\kappa}_{2,1}-\Delta \kappa\right. \\
\left.\bar{\kappa}_{2,2}-\Delta \kappa_{0,3}, \bar{\kappa}_{4,0}-\Delta \kappa, \text { two-loop }\right),
\end{aligned}
$$

from the lower relation in Fig. 5] These constraints will be used in the next subsection. We emphasise that the above relations are valid in arbitrary dimensions as the FRG relations derived in the last Section III A. In contradistinction to the FRG-relations the DSE relations depend on the dimension via the $\Delta \kappa_{2 n, m}$.

\section{Proof}

We now proceed to show that

$$
\bar{\kappa}_{2 n, m}=0
$$

is the only scaling solution of infrared Yang-Mills theory in Landau gauge. To this end we note that scaling in the ghost-DSE (28) implies that

$$
\Delta \kappa=\bar{\kappa}_{2,1} \geq 0,
$$

where we have used that the ghost-FRG entails $\bar{\kappa}_{2,1} \geq 0$. Furthermore we obtain the two constraints

$$
\bar{\kappa}_{4,0} \geq \Delta \kappa, \quad \bar{\kappa}_{2,2} \geq \Delta \kappa,
$$



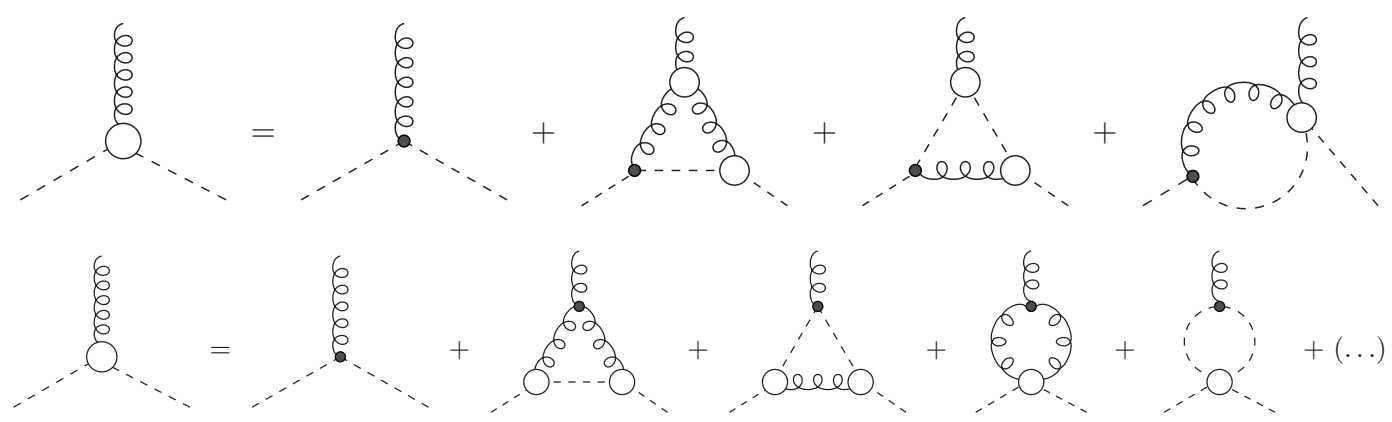

FIG. 5: Dyson-Schwinger equations for the ghost gluon vertex. Empty circles denote dressed vertex functions. All internal propagators are dressed; the corresponding filled circles have been omitted for clarity of the figures. One-loop diagrams with the same scaling behaviour are only shown once. The ellipses denotes the other one- and two-loop diagrams which are not needed for our analysis.

from the two DSEs for the ghost gluon vertex, where we use $0 \leq \bar{\kappa}_{2,1} \leq \bar{\kappa}_{2,2}-\Delta \kappa$ from (29), and $0 \leq \bar{\kappa}_{2,1} \leq$ $\bar{\kappa}_{4,0}-\Delta \kappa$ from (30). However, the ghost FRG led to the constraint (20). Together with (32) and (33) this immediately leads to

$$
\Delta \kappa=0,
$$

and therefore also $\bar{\kappa}_{2,1}=0$ due to (32).

It remains to be shown that this implies that all of the $\bar{\kappa}_{2 n, m}$ need to be zero. To this end we resort to the FRGequations. The FRG-equations for all $\bar{\kappa}_{2 n, m}$ contain at least one diagram that solely depends on $N_{2 n, m}$ ghostgluon vertices. This implies

$$
\bar{\kappa}_{2 n, m} \leq N_{2 n, m} \bar{\kappa}_{2,1}=0 .
$$

Together with (24) this leads to

$$
\bar{\kappa}_{2 n, m}=0 .
$$

Written in terms of our original anomalous dimensions $\kappa_{2 n, m}$, see (14), this implies that

$$
\kappa_{2 n, m}=\Delta d_{2 n+m}+\frac{1}{2}\left(2 n \kappa_{2,0}+m \kappa_{0,2}\right),
$$

with

$$
\Delta d_{2 n+m}=(2-2 n-m) \frac{d-4}{4},
$$

see (14) and (15) respectively. Eq. (37) and (38) represent the unique scaling solution of infrared Yang-Mills theory.

However, we would like to emphasise that the values of the anomalous scalings $\kappa_{2,0}, \kappa_{0,2}$ of the propagators cannot be fixed by scaling arguments. They have to be computed by solving the corresponding FRG and DSE equations, see e.g. [5, 66, 10, 18, 19]. Instead, there is one final piece of information which can be extracted from the scaling analysis, namely constraints on the values for $\kappa_{2,0}, \kappa_{0,2}$ : From (27) we deduce $\Delta \kappa_{0,4} \leq 0$. Using (25) we have $(4-d) / 2+2 \kappa_{0,2} \leq 0$, and with (34) we then conclude that [18, 20]

$$
\kappa_{2,0}=-\frac{4-d}{4}-\frac{1}{2} \kappa_{0,2} \geq-\frac{1}{2} \frac{4-d}{4} .
$$

Eq. (39) is in accordance with the Gribov-Zwanziger confinement scenario [2, 3] which predicts ghostenhancement.

Finally we discuss the fate of the classical terms with $\kappa_{0,2}^{\text {class }}=\kappa_{2,0}^{\text {class }}=0$. The propagator scaling stemming from the quantum fluctuations is summarised qualitatively as

$$
\kappa_{2,0} \geq-\frac{1}{2} \frac{4-d}{4}, \quad \kappa_{0,2} \leq-\frac{4-d}{4} .
$$

With $\kappa_{0,2} \leq \kappa_{0,2}^{\text {class }}=0$, the classical contribution does not change the gluon scaling. In turn, for $\kappa_{2,0}>0$, the inverse ghost propagator would be dominated by its classical part, which has to be cancelled if scaling applies. This is the adjustment of the horizon condition, see e.g. [5, 10]. Note also that the bound (40) in principle also allows for $\kappa_{2,0}<0$ for $d<4$. In practice, however, one finds $\kappa_{2,0}>0$, i.e. $\kappa_{2,0}(d=3)=0.40$, and $\kappa_{2,0}(d=2)=1 / 5$ for a classical ghost-gluon vertex [18]. For these values to drop below zero, the full ghost-gluon vertex would have to deviate drastically from the classical vertex. This is almost excluded by lattice computations [21, 22] and DSE self-consistency checks [5, 23].

Numerical solutions for the ghost and gluon DSE as well as the corresponding FRGs in agreement with Eq. (37) and (38) have been given in [10, 19, 23, 24, 25]. In 26] a truncation has been employed which effectively converts the DSEs into equations with dressed vertices resembling the structure of FRGs. This procedure allows for interesting numerical solutions. However, the vertices used are neither consistent with the (unique) infrared scaling laws Eq. (37) and (38), nor with the standard RG-scaling. At its core this is due to the fact that the system of FRG equation cannot be solved by in the IR as the gluonic vertices in 26] are too singular.

These comments complete our proof. 


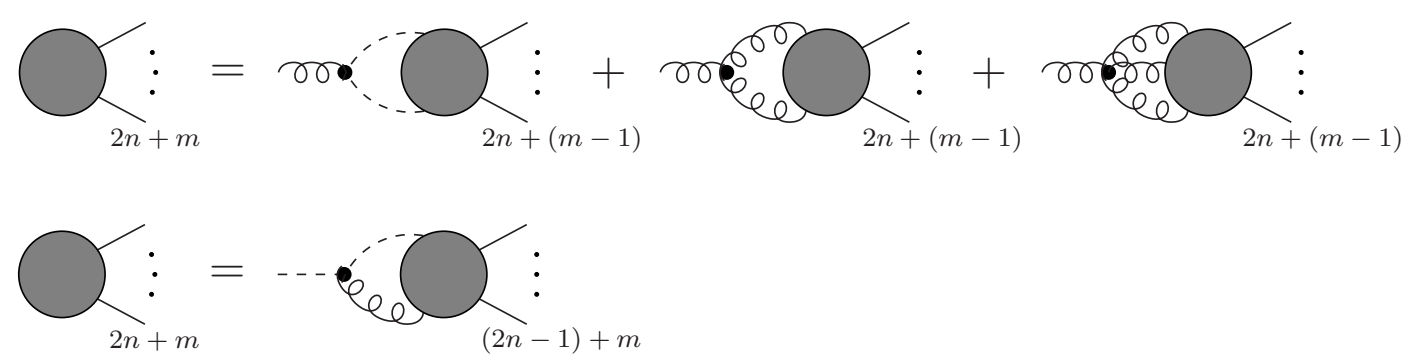

FIG. 6: General Dyson-Schwinger equations for $\Gamma^{(2 n, m)}$. The straight legs denote either ghost or gluon lines with multiplicity given below the diagram, respectively.

\section{KINEMATICS}

We have derived the unique scaling solution (37) with the assumption of conformal scaling. As already argued in [8] and in the introduction, possible conformal invariance entails that no further constraint on $\kappa_{2,0}$ and $\kappa_{0,2}$ apart from (40) can be derived from pure scaling arguments. Here we shall make this formal argument more explicit, and also discuss the question of existence of a specific scaling solution including various kinematical limits.

This is also interesting for the following reason: From our analysis in the previous section we know that the classical purely gluonic vertices are sub-dominant, and the ghost-gluon vertex has its classical global scaling. Note however that a ghost gluon vertex with $\bar{\Gamma}^{(2,1)} \simeq S^{(2,1)}$ requires the cancellation of the classical term in the DSE and the initial condition in the FRG equations in some kinematical limits, i.e. vanishing ghost or anti-ghost momentum. In these limits the diagrammatical scaling would then be dominated by the classical part/initial condition.

As already discussed above, the FRG equations cannot provide constraints on $\kappa_{2,0}, \kappa_{0,2}$, as the $\kappa$ 's drop out of the FRG-scaling analysis after employing the parameterisation (4). The FRG-diagrams then reduce to one loop diagrams with bare propagators and dressed vertices $\bar{\Gamma}^{(2 n, m)}$ with only canonical global scaling due to (36). Here and in the following we use the term 'global scaling' when all external momenta scale in contrast to 'kinematical scaling' when only some external momenta are involved ${ }^{1}$. Note that the $\bar{\Gamma}^{(2 n, m)}$ carry kinematical singularities as is well-known from perturbation theory. In Landau gauge these may be meliorated by transversality. Since we are working in Landau gauge we are only interested in transversal contributions to all vertices; in Landau gauge the set of fully transversal vertices together with the ghost propagator and transversal gluon propagator provide a closed set of FRG and DSE equations. In turn, vertices with at least one longitudinal gluon satisfy

\footnotetext{
1 This corresponds to 'uniform' vs. 'collinear' singularities in the terminology of [15].
}

DSEs and FRGs that also depend on the transversal vertices and only on the transversal gluon propagator, see also [10].

For these reasons we concentrate on the purely transversal part of the vertices, $\Gamma_{\perp}(2 n, m)$. For the treatment of kinematical scaling we also extend our notation to

$$
\begin{gathered}
\Gamma_{\perp}{ }^{(2 n, m)}\left(\lambda \vec{p}_{1, r}, \vec{p}_{r+1,2 n}, \lambda \vec{p}_{2 n+1,2 n+s}, \vec{p}_{2 n+s+1,2 n+m}\right) \\
\stackrel{\lambda \rightarrow 0}{\longrightarrow} \lambda^{2\left(d_{r+s}+\kappa_{2 n, m}^{r, s}\right)} \Gamma_{\perp r, s}^{(2 n, m)}(\vec{p})
\end{gathered}
$$

where $\Gamma_{\perp}{ }_{r, s}^{(2 n, m)}$ stands for the infrared leading term, $\vec{p}_{i, j}=\left(p_{i}, \ldots, p_{j}\right)$, and $\vec{p}$ is not exceptional by itself. Here, $s$ counts scaling gluon momenta, and $r=r_{1}+r_{2}$ counts $r_{1}$ scaling ghost momenta and $r_{2}$ scaling anti-ghost momenta. Analogously to (12) the total scaling $\lambda^{2 t_{2 n, m}^{r, s}}$ of the full vertex $\Gamma^{2 n, m}$ reads

$$
t_{2 n, m}^{r, s}=d_{r+s}+\kappa_{2 n, m}^{r, s}
$$

The global scaling (37) is reproduced by

$$
\kappa_{2 n, m}^{r, s}=\kappa_{2 n, m}, \quad \forall r+s=2 n+m-1,
$$

due to momentum conservation.

In the following we shall show that a dressed ghostgluon vertex without kinematic singularities is a possible solution of the DSE and FRG systems. In this case the scaling relations for the ghost-gluon vertex $\Gamma_{\perp}{ }^{(2,1)}$ are

$$
\kappa_{2,1}^{1,0}=\kappa_{2,1}^{0,1}=\kappa_{2,1}=0 .
$$

A ghost-gluon vertex with (44) reads

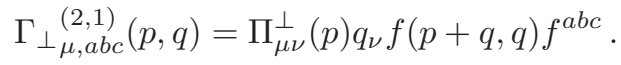

where

$$
\Pi_{\mu \nu}^{\perp}(p)=\delta_{\mu \nu}-\frac{p_{\mu} p_{\nu}}{p^{2}}
$$

and $f$ is a non-singular function of both momenta. Here $p$ is the gluon momentum, $q+p$ is the ghost momentum 


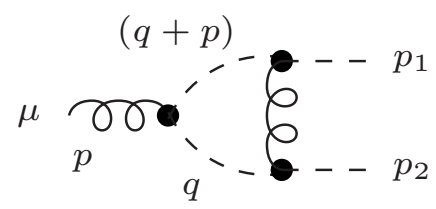

FIG. 7: Infrared leading diagram in the DSE for the ghostgluon vertex.

and $q$ is the anti-ghost momentum respectively. Ghostanti-ghost symmetry is implicit with

$$
\Pi_{\mu \nu}^{\perp}(p) q_{\nu}=\Pi_{\mu \nu}^{\perp}(p)(p+q)_{\mu}, \quad f(p+q, q)=f(q, p+q) .
$$

Now we assume that the leading infrared parts of all vertices are already provided by diagrams only depending on ghost-gluon vertices. This entails that, with the exception of the ghost-gluon vertex, all ghost or anti-ghost legs of arbitrary vertices are proportional to linear powers of the corresponding external momentum. The reason is that each of these legs is attached to an internal transversal gluon. With (47) we then can always rewrite this diagram as proportional to linear powers of the external momenta.

With this fact in mind we can deduce the scaling from the general form of the vertex-DSEs. Diagrammatically they are depicted in Fig. 6. We begin our analysis with vertices where one external gluon momentum is vanishing. To this end we take the external momentum $p=p_{\text {bare }}$ of the bare vertex to zero by keeping the other momenta at non-exceptional values. Extracting the linear momenta assigned to the internal ghost lines and projecting onto transverse components we get

$$
\begin{aligned}
& \Pi_{\mu \nu}^{\perp}(p) \int d q q^{d-1} \int d \Omega_{q} \frac{1}{q^{2(1+\kappa)}} \frac{1}{(q+p)^{2(1+\kappa)}} \\
& \quad \times q_{\nu}(q+p)_{\rho} q_{\sigma} \mathcal{I}_{\rho \sigma \mu_{1} \cdots \mu_{m-1}}\left(p, q, p_{1} \cdots p_{2 n+m-1}\right)
\end{aligned}
$$

where $\int d \Omega_{q}$ stands for the angular integration stemming from the $q$-integration, and $\mu=\mu_{m}$.

As an example we discuss the simplest possible diagram for the case of no further external gluons, $m=1$, shown in Fig. 7 with a similar momentum routing as in Eq. (48). In this diagram each of the three ghostgluon vertices generates one four-momentum. Due to the transversality of the involved two gluon lines these can be converted into the factor $q_{\nu}(q+p)_{\rho} q_{\sigma}$ also appearing in Eq. (48). For a non-vanishing integral we need a further power in $q$, otherwise the integral is proportional to $p_{\nu}$ and vanishes due to projection with $\Pi_{\mu \nu}^{\perp}(p)$. Indeed an extra factor $p_{i} \cdot q$ for some $i=1, \ldots, 2$ is generated by the internal gluon. In the general expression (48) this extra factor is provided by the kernel $\mathcal{I}$. Counting powers of the scaling momenta $p$ and $q$ we find

$$
\kappa_{2 n, 1}^{0,1}=\frac{d}{2}-2 \kappa
$$

for $m=1$. From (48) we have $d / 2$ from the integration, -2 from the two denominators of the propagators, $-2 \kappa$ from the two ghost dressing functions in the propagators and +2 from the four powers of $q$. The other momenta, $p_{1}$ and $p_{2}$ do not scale. The meaning of Eq. (49) is the following: we only obtain an infrared dominated, divergent and therefore scaling integral for $\kappa>d / 4$, otherwise we cannot say something definite from the scaling analysis. Whether the integral is then vanishing or finite depends on the details of the kernel $\mathcal{I}$ and the angular integration; consequently the integral may or may not display kinematical scaling for vanishing gluon momentum in this case.

On the other hand, in the general case where further external gluons are present, i.e. $m>1$, we have contractions of $q_{\nu}(q+p)_{\rho} q_{\sigma}$ with $\mathcal{I}_{\rho \sigma \mu_{2} \cdots \mu_{m}}\left(p, q, l_{2} \cdots l_{2 n+m}\right)$. These contractions generate terms proportional to $p_{\mu_{i}}$ with $i=1, \ldots,(m-1)$ that do not vanish even when all external gluon legs are transversally projected. Thus we can have terms in the integrand proportional to $q_{\nu} p_{\rho} q_{\sigma}$. In order to decide whether the integral produces kinematical singularities or not we count powers in scaling loop momenta, i.e. two factors of $q$, two ghost dressing functions, two denominators and the integration. We arrive at the condition

$$
\frac{d-2}{2}-2 \kappa<0 \quad \Rightarrow \quad \kappa>\frac{d-2}{4} .
$$

Thus kinematical singularities only occur for $\kappa>(d-$ $2) / 4$. However, for the scaling of the vertex in terms of the external gluon momentum we have to count all powers of scaling momenta $q$ and $p$ and arrive at ${ }^{2}$

$$
\kappa_{2 n, m}^{0,1}=\frac{d-1}{2}-2 \kappa,
$$

for $m>1$, in agreement with [15] for $d=4$.

From Eqs. (49) and (51) we observe that the kinematical gluonic singularities are smaller or equal to the lowest possible global singularity $-\kappa$ as long as $\kappa \leq(d-1) / 2$, i.e.

$$
\kappa_{2 n, m}^{0,1} \geq-\kappa \rightarrow \kappa \leq \frac{d-1}{2}
$$

Consequently the gluonic vertex dressing in the scattering kernels in Fig. 6] does not lead to divergences for $p \rightarrow 0$ in neither the gluonic nor the ghost DSEs. In particular this entails that

$$
\kappa_{2 n, m}^{1,0} \geq 0
$$

\footnotetext{
2 Since we also count powers of $p_{\mu_{i}}$ this relation possibly involves the tensor structure of the vertex $\Gamma_{2 n, m}$. The only case where this is important and interesting is the special case of the threegluon vertex $\Gamma_{0,3}$. It is easy to see that the tensor structure of the bare three gluon vertex cannot have kinematical singularities and therefore cannot take part in kinematical scaling. Since Eq. (51), however, involves an explicit scaling momentum with external Lorentz-index it has to represent the kinematical scaling of a different tensor structure of the three-gluon vertex.
} 
We conclude our analysis with a brief discussion of higher kinematical singularities, which is worked out in more detail in Appendix B There we derive the scaling relation

$$
t_{2 n, m}^{r, s}=t_{r, s}+\left\{\begin{array}{lc}
-\frac{d-2}{4}+\frac{1}{2} \kappa & r_{2}=r_{1}+1, \\
\min \left(-\kappa, \frac{2-d}{2}+2 \kappa\right) & \text { else }
\end{array}\right.
$$

To summarise: for $\kappa \leq(d-1) / 2$ we have obtained kinematical divergencies that are small enough such that they cannot invalidate the global scaling relation (37). Note however that the bound for the existence of a Fourier transform of the ghost propagator is $\kappa<(d-2) / 2$, see also [5]. If $\kappa$ exceeds $(d-2) / 2$, the ghost propagator in position space cannot be understood anymore as tempered distributions which is a necessary condition for correlation functions in a local quantum field theory. Hence the above bound $\kappa \leq(d-1) / 2$ is not relevant for this physically interesting case. We conclude that the relation (54) for the kinematical scaling together with the global scaling Eq. (37) is a possible solution of the tower of DSEs and FRGs.

We do not want to further this discussion, in particular as the actual numerical -and physically sensible- solution satisfy all of the above bounds, that is $\kappa_{\text {num }}<(d-2) / 2$. We merely would like to mention that even in the case $\kappa>(d-1) / 2$ one cannot conclude that the system is illdefined. It only means that such an anomalous scaling cannot be captured by the DSE tower of equations, where the single diagrams do not entail the full RG-scaling, and hence also do not have manifest scale invariance in the scaling region. A consistent solution to such a system necessarily requires non-trivial cancellations between different diagrams. In the ghost propagator DSE, where such cancellations cannot occur, they are not necessary; the ghost-DSE is well-defined even for $\kappa>(d-1) / 2$ in the case of full kinematical scaling of the ghost-gluon vertex.

From our kinematical analysis we find that in the case of $\kappa>(d-2) / 2$ all vertices have kinematic singularities. As stated above the DSEs cannot be used anymore. The FRGs, however, can straightaway be solved by $\bar{\kappa}_{2 n, m}^{r, s}=0$. This is also the reason why the above solution, Eq. (54), for kinematical scaling is not unique in contradistinction to the global scaling relation Eq. (37). These comments complete our proof of existence.

\section{SUMMARY}

In this work we demonstrated that there is only one, unique global scaling solution of infrared Yang-Mills theory in Landau gauge. To this end we introduced a parameterisation of the one-particle irreducible Green func- tions of the theory that splits off the renormalisation group (RG)-scaling and reduces the discussion to that of the scaling properties of RG-invariant quantities. To our mind this greatly simplifies the proof as compared to our previous work Ref. [8]. We wish to emphasise again, that the values of the anomalous scalings $\kappa_{2,0}, \kappa_{0,2}$ of the propagators cannot be fixed by scaling arguments. These have to be calculated explicitly from the corresponding FRG and DSE equations.

In addition we also demonstrated the existence of a specific scaling solution that includes 'kinematical scaling' in various kinematical limits. In contradistinction to the global scaling relation, kinematical scaling is not unique.

In general, the method presented here is also applicable to other theories. As an example we shortly discussed its applicability to scalar quantum field theories in the introduction; in [8] we discussed scaling in the gauge-Higgs theory. These applications may also be extended to Yang-Mills theories in other gauges.

\section{Acknowledgments}

We are grateful for many discussions with Reinhard Alkofer, Markus Huber, Felipe Llanes-Estrada, Kai Schwenzer and Lorenz von Smekal. C. F. was supported by the Helmholtz-University Young Investigator Grant number VH-NG-332 and J. M. P. by Helmholtz Alliance HA216/EMMI.

\section{APPENDIX A: INTEGRATED FLOW EQUATION}

The standard form in the flow equation is depicted in Fig 8 where $t=\ln k$ is the logarithmic infrared scale

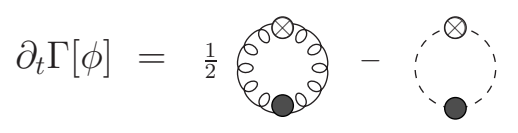

FIG. 8: Functional renormalisation group equation (FRG) for the effective action. Filled circles denote fully dressed field dependent propagators. Crosses denote the regulator insertion $\partial_{t} R$.

and the cross denotes the regulator insertion $\partial_{t} R$. If $R_{k}$ is chosen as a mode cut-off and simply removes one momentum mode from the theory, the loop integrations in the FRG equations for vertices is reduced to a single loop momentum $q^{2} \simeq k^{2}$ and we can explicitly apply the infrared power counting. This has been done in [8]. For the sake of the comparison with the DSE is also convenient to rewrite the FRG as follows [16], 


$$
\partial_{t} \Gamma[\phi]=\frac{1}{2} \operatorname{Tr}\left(\frac{1}{\Gamma^{(2)}[\phi]+R} \partial_{t} R\right)=\frac{1}{2} \operatorname{Tr} \partial_{t} \ln \left(\Gamma^{(2)}[\phi]+R\right)-\frac{1}{2} \operatorname{Tr}\left(\frac{1}{\Gamma^{(2)}[\phi]+R} \partial_{t} \Gamma^{(2)}\right) .
$$

Upon integration from an initial momentum scale $k=\Lambda$ to $k=0$ this yields

$$
\Gamma[\phi]=S_{\Lambda}[\phi]+\left(\frac{1}{2} \operatorname{Tr} \ln \Gamma^{(2)}[\phi]+\text { ren }\right)-\frac{1}{2} \int_{\Lambda}^{0} \frac{d k^{\prime}}{k^{\prime}} \operatorname{Tr}\left(\frac{1}{\Gamma^{(2)}[\phi]+R^{\prime}} \partial_{t^{\prime}} \Gamma^{(2)}\right)+\text { ren },
$$

where $S_{\Lambda}=\left(\Gamma_{\Lambda}-\operatorname{Tr} \ln \left(\Gamma^{(2)}[\phi]+R\right)_{\Lambda}-\right.$ ren $)$ entails the initial condition at $k=\Lambda$ and the integral term on the rhs of $\mathrm{A} 2$ is an RG-improvement term. If we perform a momentum rescaling as in (8) including that of $\Lambda: \Lambda \rightarrow$ $\lambda \Lambda$, the last term shows at most the same scaling as the first one. This entails up to renormalisation, that

$$
\lim _{\lambda \rightarrow 0} \Gamma[\phi] \simeq \frac{1}{2} \operatorname{Tr} \ln \Gamma^{(2)}[\phi],
$$

as far as infrared scaling is concerned. Taking a gluon or ghost derivative of A3 leads to the diagrammatical representation of the infrared asymptotics of the flow in Fig. 1.

\section{APPENDIX B: VERTICES WITH TWO OR MORE EXTERNAL SCALING LEGS}

In this appendix we investigate the kinematical scaling for vertices $\Gamma^{2 n, m}$ with $s$ soft gluon lines, $r_{1}$ soft ghost lines and $r_{2}$ soft anti-ghost lines with $r=r_{1}+r_{2}$. Due to ghost-anti-ghost symmetry we can always choose $r_{2} \geq$ $r_{1}$. We will explore some general situations and discuss exceptions at the end of this section.

First we discuss diagrams with isolated external legs with vanishing momenta, i.e. diagrams where these legs are not neighbouring. These are simple. In the case of isolated external ghost lines no divergences are encountered due to Eq. (53). Diagrams with isolated external gluon lines scale like the corresponding diagrams with one vanishing momentum, i.e. Eq. (49) if no hard external gluon lines are present and Eq. (51) otherwise. This can be easily verified by going through some explicit examples. In case some of the external gluonic momenta are parallel we always find Eq. (51); the argument is similar to that given below Eq. (51). Having said this, we concentrate on vertices with only neighbouring legs with vanishing momenta for the remainder of this section.

In the following argument we concentrate on the FRG diagrams which are only one loop. A part of the related diagrams decay into three classes depicted in Fig. 9, The other diagrams involve vertices with soft and hard parts that cannot be separated in the above way. We will evaluate these diagrams at the end of our discussion. The first two classes of diagrams in Fig. 9] summarise possible diagrams of neighbouring vanishing legs for $r_{2}=r_{1}$ or $r_{2}=r_{1}+2$. The third class summarises those with $r_{2}=r_{1}+1$. The total scaling of a vertex with $r_{1}$ ghosts

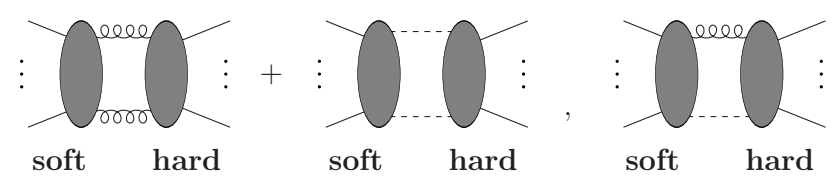

FIG. 9: Diagram classes with kinematical scaling of only part of the vertices.

and $r_{2}$ anti-ghosts with $r=r_{1}+r_{2}$ and $s$ gluons is given by

$$
t_{r, s}=d_{r+s}+\frac{1}{2}\left(r \kappa_{2,0}+s \kappa_{0,2}\right)
$$

where we have extended the scaling for ghost gluon vertices with $r_{1}=r_{2}$ to non-existing vertices with $r_{1} \neq r_{2}$. We are now in the position to deduce the scaling of vertices $\Gamma^{2 n, m}$ with $s+r_{1}+r_{2}$ vanishing external momenta for gluons+ghosts+anti-ghosts respectively, by relating it to the global scaling (B1) of a vertex with only vanishing external momenta: we simply remove the hard parts of the diagrams and close the remaining open lines with some combinations of soft vertices and propagators, the scaling of which we then subtract. What is left is the scaling $t_{2 n, m}^{r, s}$ of the diagrams in Fig. 9. Since this procedure is insensitive to the specific combination of vertices added, we simply take a minimal number of additional soft vertices for the explicit computations, without loss of generality.

In the first diagram in Fig. 9] we substitute the hard part of the diagram by a soft full three gluon vertex, hence adding a further vanishing gluon momentum. From the resulting global scaling of $t_{r, s+1}$ we have to subtract the global scaling $t_{0,3}$ of the three-gluon vertex leading to

$$
t_{2 n, m}^{r, s} \leq t_{r, s+1}-t_{0,3}=t_{r, s}+\frac{2-d}{2}+2 \kappa .
$$

In (B2) we have used that $d_{r+s+1}-d_{3}=d_{r+s}-1$. As already pointed out above, e.g. substituting the hard part of the diagram with two ghost gluon vertices and one connecting ghost propagator leads to the same result. The same is true for any other combination of vertices in the hard part of the diagram. The $\leq$ in Eq. (B2) expresses 
the fact that there are cases where the kinematical situation of the same vertex may be represented by the first or second digram of Fig. 9 and either one may carry the leading kinematical singularity.

To analyse the second diagram we have to distinguish two cases. For $r_{1}=r_{2}$ we substitute the hard part by a soft full ghost gluon vertex, hence adding a further vanishing ghost momentum. Apart from subtracting its global scaling $t_{2,1}=1 / 2$, we also have to take care of the kinematical intricacies of ghost lines. The hard part of the second class of diagrams in Fig. 9] also carries a momentum dependence that is linear in the momenta of the soft ghost legs. This has to be added separately, and we arrive at

$$
t_{2 n, m}^{r, s} \leq t_{r, s+1}+1-t_{2,1}=t_{r, s}-\kappa
$$

where we have used that $d_{r+s+1}=d_{r+s}-(d-2) / 4$. If $r_{1}=r_{2}-2$ the two connecting lines between the soft and the hard part of the diagram are two anti-ghosts and we have at least to substitute the hard part of the diagram with two ghost gluon vertices and one connecting gluon propagator. In our counting we again have to add two linear powers of momenta due to the two connecting ghost legs. The net result is the same as for the case $r_{1}=r_{2}$. Therefore the result Eq. (B3) with $r=r_{1}+r_{2}$ summarises both cases.

In the third diagram we substitute the hard part of the diagram by a soft full ghost gluon vertex, hence adding a further vanishing ghost momentum. Apart from subtracting its global scaling $t_{2,1}=1 / 2$, the hard part of the third class of diagrams in Fig. 9] also carries a momentum dependence that is linear in the momentum of the soft ghost leg which has to be added separately. We are led to

$$
t_{2 n, m}^{r, s}=t_{r+1, s}+\frac{1}{2}-t_{2,1}=t_{r, s}-\frac{d-2}{4}+\frac{1}{2} \kappa .
$$

Note that $t_{2 n, m}^{r, s}=t_{r+1, s}$ with $r+1=2 n$.

We summarise the results of this section with the scaling relation

$$
t_{2 n, m}^{r, s}=t_{r, s}+\left\{\begin{array}{lc}
-\frac{d-2}{4}+\frac{1}{2} \kappa & r_{2}=r_{1}+1 \\
\min \left(-\kappa, \frac{2-d}{2}+2 \kappa\right) & \text { else }
\end{array}\right.
$$

where we have used (B2), (B3) and (B4). Note that one can swap between the different classes of diagrams by isolating one or two soft ghost or anti-ghost vertices within the hard part of the diagrams. This effectively removes these vertices from the counting. However, since the scaling relations is different for the three classes it could pay off in a more singular behaviour. It is easy to check that this is not the case, and hence (B5) represents the full maximal scaling of the diagram classes depicted in Fig. 9.

We close with a discussion of the remaining diagram classes. A interesting specific case is depicted in Fig. 10. This seems to entail that one can have neighbouring ghost vertices or anti-ghost vertices. However, one can show that this only is possible at the expense of additional loops as is present in Fig. 10. At its core this relates to the fact that the ghost number of vertices $\Gamma^{2 n, m}$ vanishes. The scaling of the related sub-diagram is always positive, in the present case it is $1 / 2$. Restricting ourselves again to the case $r_{1} \leq r_{2}$, we deduce that for $r_{2}>r_{1}+2$ we are effectively reduced to the soft scaling of a diagram with $r_{1}$ ghost and $r_{1}+2$ anti-ghost legs and hence Eq. (B5) applies. Because of ghost-anti-ghost symmetry this covers the general case.

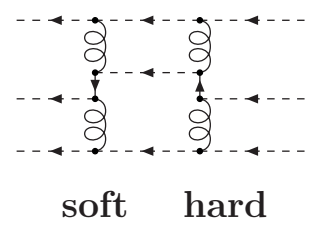

FIG. 10: Example from an exceptional class of diagrams with kinematical scaling of only part of the vertices.
[1] T. Kugo and I. Ojima, Prog. Theor. Phys. Suppl. 66, 1 (1979) [Erratum Prog. Theor. Phys. 71, 1121 (1984)]; T. Kugo, arXiv:hep-th/9511033.

[2] V. N. Gribov, Nucl. Phys. B 139, 1 (1978).

[3] D. Zwanziger, Phys. Lett. B 257, 168 (1991); Nucl. Phys. B 364, 127 (1991); Nucl. Phys. B 412, 657 (1994); Phys. Rev. D 65, 094039 (2002) [arXiv: hep-th/0109224].

[4] L. von Smekal, A. Hauck and R. Alkofer, Annals Phys. 267 (1998) 1 [Erratum-ibid. 269 (1998) 182]
arXiv:hep-ph/9707327; L. von Smekal, R. Alkofer and A. Hauck, Phys. Rev. Lett. 79 (1997) 3591 arXiv:hep-ph/9705242.

[5] C. Lerche and L. von Smekal, Phys. Rev. D 65, 125006 (2002) arXiv:hep-ph/0202194.

[6] J. M. Pawlowski, D. F. Litim, S. Nedelko, and L. von Smekal Phys. Rev. Lett. 93 (2004) 152002 hep-th/0312324; AIP Conf. Proc. 756 (2005) 278 hep-th/0412326. 
[7] R. Alkofer, C. S. Fischer and F. J. Llanes-Estrada, Phys. Lett. B 611, 279 (2005) arXiv:hep-th/0412330.

[8] C. S. Fischer and J. M. Pawlowski, Phys. Rev. D 75 (2007) 025012 arXiv:hep-th/0609009.

[9] M. Q. Huber, R. Alkofer, C. S. Fischer and K. Schwenzer, Phys. Lett. B 659 (2008) 434 arXiv:0705.3809 [hep-ph]].

[10] C. S. Fischer, A. Maas and J. M. Pawlowski, arXiv:0810.1987 [hep-ph].

[11] J. M. Cornwall, Phys. Rev. D 26 (1982) 1453.

[12] A. C. Aguilar, D. Binosi and J. Papavassiliou, Phys. Rev. D 78, 025010 (2008) arXiv:0802.1870 [hep-ph]].

[13] Ph. Boucaud, J. P. Leroy, A. L. Yaouanc, J. Micheli, O. Pene and J. Rodriguez-Quintero, JHEP 0806, 012 (2008) arXiv:0801.2721 [hep-ph]].

[14] D. Dudal, J. A. Gracey, S. P. Sorella, N. Vandersickel and H. Verschelde, arXiv:0806.4348 [hep-th].

[15] R. Alkofer, M. Q. Huber and K. Schwenzer, arXiv:0801.2762 [hep-th]; arXiv:0812.4045 [hep-ph].

[16] J. M. Pawlowski, Annals Phys. 322 (2007) 2831 arXiv:hep-th/0512261.

[17] J. M. Pawlowski, Int. J. Mod. Phys. A 16 (2001) 2105.
[18] D. Zwanziger, Phys. Rev. D $65 \quad$ (2002) 094039 arXiv:hep-th/0109224.

[19] C. S. Fischer and R. Alkofer, Phys. Lett. B 536 (2002) 177 arXiv:hep-ph/0202202.

[20] A. Maas, J. Wambach, B. Gruter and R. Alkofer, Eur. Phys. J. C 37 (2004) 335 arXiv:hep-ph/0408074.

[21] E. M. Ilgenfritz, M. Muller-Preussker, A. Sternbeck, A. Schiller and I. L. Bogolubsky, Braz. J. Phys. 37, 193 (2007) arXiv:hep-lat/0609043.

[22] A. Cucchieri, A. Maas and T. Mendes, Phys. Rev. D 77, 094510 (2008) arXiv:0803.1798 [hep-lat]].

[23] W. Schleifenbaum, A. Maas, J. Wambach and R. Alkofer, Phys. Rev. D 72, 014017 (2005) arXiv:hep-ph/0411052.

[24] C. S. Fischer and H. Gies, JHEP 0410, 048 (2004) arXiv:hep-ph/0408089.

[25] C. Kellermann and C. S. Fischer, Phys. Rev. D 78, 025015 (2008) arXiv:0801.2697 [hep-ph]].

[26] J. C. R. Bloch, Few Body Syst. 33, 111 (2003) arXiv:hep-ph/0303125. 